\title{
Predicting individual differences in motor learning: a critical review
}

\author{
Rajiv Ranganathan ${ }^{1,2}$, Simon Cone', Brian Fox'
}

\begin{abstract}
The ability to predict individual differences in motor learning has significant implications from both theoretical and applied perspectives. However, there is high variability in the methodological and analytical strategies employed as evidence for such predictions. Here, we critically examine the evidence for predictions of individual differences in motor learning by reviewing the literature from a 20-year period (2000-2020). Specifically, we examined four factors: (i) the predictor and predicted variables used, (ii) the strength of the prediction and associated sample size, (iii) the timescale over which the prediction was made, and (iv) the type of motor task used. Overall, the results highlight several issues that raise concerns about the quality of the evidence for such predictions. First, there was a large variation in both predictor and predicted variables, suggesting the presence of a large number of researcher degrees of freedom. Second, sample sizes tended to be small and the strength of the correlation showed an inverse relation with sample size. Third, the timescale of most predictions was very short, mostly constrained to a single day. Last, most studies were largely restricted to two experimental paradigms - adaptation and sequence learning. Based on these issues, we highlight recommendations for future studies to improve the quality of evidence for predicting individual differences in motor learning.
\end{abstract}

\section{Keywords}

correlation, skill, power, sample size, pre-registration

'Department of Kinesiology, Michigan State University, East Lansing, MI, USA

${ }^{2}$ Deparment of Mechanical Engineering, Michigan State University, East Lansing, MI, USA

Corresponding Author:

Rajiv Ranganathan, Department of Kinesiology, 308 W Circle Dr East Lansing MI USA 48824 rrangana@msu.edu 


\section{Introduction}

It has been long recognized that individuals who undergo the same training show differences in how much they learn during practice. These individual differences in motor learning have been the centerpiece of several long-standing theoretical issues ranging from general motor abilities (Seashore, 1930), aptitude-treatment interactions (Cronbach, 1957) and cognitive-intellectual determinants (Ackerman, 1988). Several reviews have focused on this issue (Marteniuk, 1974; Ackerman, 1987; Adams, 1987; Seidler and Carson, 2017), including a recent historical perspective (Anderson et al., 202I).

An important issue in this regard is whether these individual differences can be predicted - i.e., whether it is possible to identify how much an individual will learn based on measurements either early on or even prior to the practice period. Here, we use the term 'prediction' to refer to contexts where the independent variable is measured earlier in time relative to the measurement of the dependent variable. Making these predictions is not only important for testing theoretical ideas of individual differences but is also critical for applied domains like coaching and rehabilitation, where the ability to predict learning could have significant implications for the design and implementation of practice strategies.

However, a challenge in synthesizing the evidence for predicting individual differences is the variability in the methodological and analytical strategies employed. These variations include differences in the types of variables measured, the time point in learning when these variables are measured, and the types of motor tasks chosen. These variations are not only important from a methodological viewpoint but also have theoretical implications as they relate to different issues in motor learning. For example, predictions made using variables measured during practice (i.e., performance 'inside' the same task) are relevant to the theoretical construct of 'task specificity' (Henry, 1968) since future performance in a task is being predicted from prior performance on the same task. On the other hand, predictions made using variables measured before practice (i.e., performance 'outside' the task) provide insight into whether there are general motor learning abilities that underlie learning (Seashore, 1930; Hands et al., 2018). Therefore, it is important to assess the variability in these choices when examining the strength of the evidence for prediction of individual differences.

The purpose of this review was to critically examine the evidence for predictions of individual differences in motor learning over a 20-year period (from 2000-2020). We focused on four primary factors - (i) the type of predictor and predicted variables, (ii) the strength of the prediction, (iii) the timescale of the prediction, and (iv) the type of task used. By analyzing these factors, we identify challenges in the current state of evidence and suggest directions for future work.

\section{Methods}

We examined studies published between 2000-2020 for the purpose of this review. We screened studies based on the following criteria: (i) the study focused on motor learning in unimpaired individuals, (ii) the study had a 'prediction' (i.e., the independent variable was measured ahead of time relative to the dependent variable), (iii) the prediction was based on a single independent variable, and (iv) the prediction was quantified in terms in terms of a Pearson correlation coefficient. The justification for excluding studies using a 'group differences' approach (e.g., using an ANOVA to compare 'good' vs. 
'poor' learners) was based on the observation that these studies typically examined groups posthoc by dichotomizing a continuous variable, which can be misleading (MacCallum et al., 2002; Altman and Royston, 2006). To capture a wide range of articles, our search strategy involved the use of Web of science and PubMed (with the keywords "motor learning", "individual differences" and "prediction"). We also identified papers from references cited from selected articles. If a paper reported multiple experiments, each experiment was considered as an independent study. Overall, we selected 30 papers with a total of 37 studies for the purpose of this review.

\section{Variables involved in prediction}

Based on the correlation that was reported, we coded the predictor variable (i.e., the variable that was used to predict learning) and the predicted variable (i.e., the measure of learning) in each study. The predictor variable was coded based on two factors: (i) its relation to the task being learned - i.e., whether it was "inside" or "outside" the task being learned, and (ii) the type of variable - i.e., whether it was based directly on a neural measure (e.g., EEG, fMRI) or a behavioral measure.

The predicted variable was coded based on the learning measure, which we categorized into three types (i) a 'learning level' (e.g., the movement time at the end of practice), (ii) a 'change score' (e.g., the difference between movement times in the first and last block of practice) or (iii) a 'learning rate' (e.g., the exponent of an exponential fit of the movement time data across practice). Furthermore, we also examined how these learning measures were computed - e.g., if a change score was reported, we also noted if this was reported as an absolute change or a percentage change. Similarly, if learning rates were computed, we noted if this was done using a single exponential, a double exponential, a power law, or another method (Newell et al., 2006).

\section{Strength of prediction}

The strength of prediction in each study was quantified using the Pearson correlation coefficient. Because some papers had multiple correlations reported, we used two criteria to select the specific correlation- (i) because the focus of the study was on the distinction between behavioral and neural variables, we excluded correlations with demographic variables (such as age), and (ii) of the remaining correlations, we chose the one with the strongest correlation (i.e., highest absolute value of the correlation coefficient).

Because the focus of the study was only on the strength of the correlation (and not the sign), we used the absolute value of the correlation (Vul and Pashler, 2017) to compare different studies. If an $\mathrm{R}^{2}$ value was directly reported using a linear regression, we transformed it to the absolute value of the Pearson correlation by taking the square root. In addition to the absolute value of the correlation, the sample size that was used in the correlation analysis was also noted.

\section{Time scale of prediction}

We examined the time scale of prediction - i.e., the time gap in 'real-world' units (measured in terms of days) between when the predictor variable was measured and when the predicted variable was 
measured. For example, if both predictor and predicted variable were measured on the same day, the time scale of prediction was reported as I day.

\section{Type of motor task}

We categorized the type of motor task used in each study. The classification of tasks was done based on six categories- adaptation, applied, coordination, sequence, tracking and variability (Ranganathan et al., 202I)

\section{Results}

\section{Variables involved in prediction}

\section{Predicted variables}

There was a wide variation in types of predicted variables (Fig IA). Learning rates $(n=15,40.5 \%)$ and learning levels $(n=15,40.5 \%)$ were more common compared to change scores $(n=7,19 \%)$. In addition, even within each category there were differences in how they were operationalized. For example, for the 15 studies that measured 'rate of learning', this was operationalized using single exponential fits $(n=4)$, power laws $(n=3)$, average of a specified number of trials $(n=3)$, state space models $(n=2)$, linear slopes $(n=2)$ and a time to criterion measure $(n=1)$. Similarly for the 7 studies using a change score, this was operationalized both as an absolute change score $(n=4)$ and a relative change score $(n=3)$.

\section{Predictor variables}

There was a wide variation in types of predicted variables (Fig IB). The use of 'outside' task predictors $(n=33,89.2 \%)$ was more common relative to those 'inside' tasks $(n=4,10.8 \%)$. There was a roughly even split between studies using neural $(n=19,51.4 \%)$ and behavioral predictor variables $(n$ $=18,48.6 \%)(\mathrm{Fig} \mid \mathrm{A})$. Analysis of the predictors (Fig IC) indicated that the most common neural predictors were related to structural connectivity as indexed through fractional anistropy $(n=5)$ and measures of functional connectivity either measured through fMRI $(n=5)$ or EEG $(n=4)$. The most common behavioral predictors were motor variability $(n=9)$ and working memory $(n=4)$. 
A

Learning level (41\%)

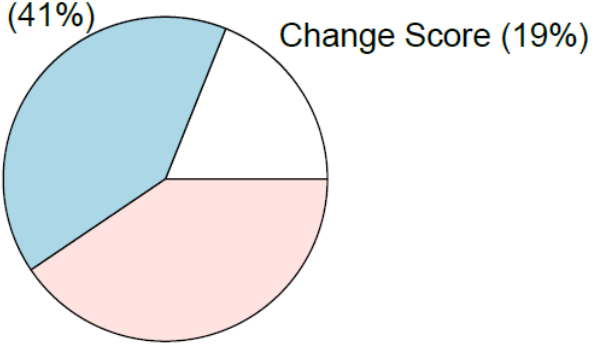

Learning rate $(41 \%)$
B

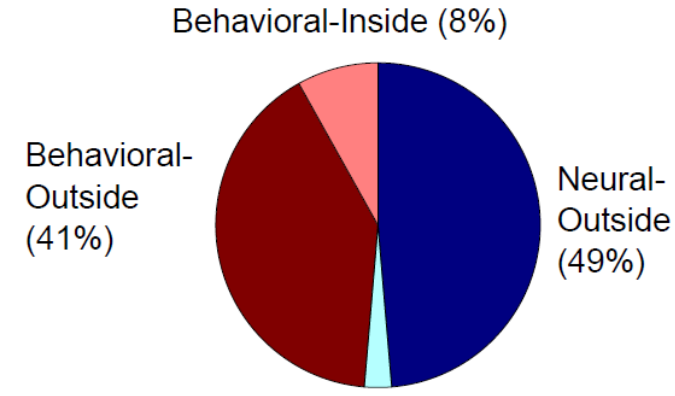

Neural-Inside (3\%)

C

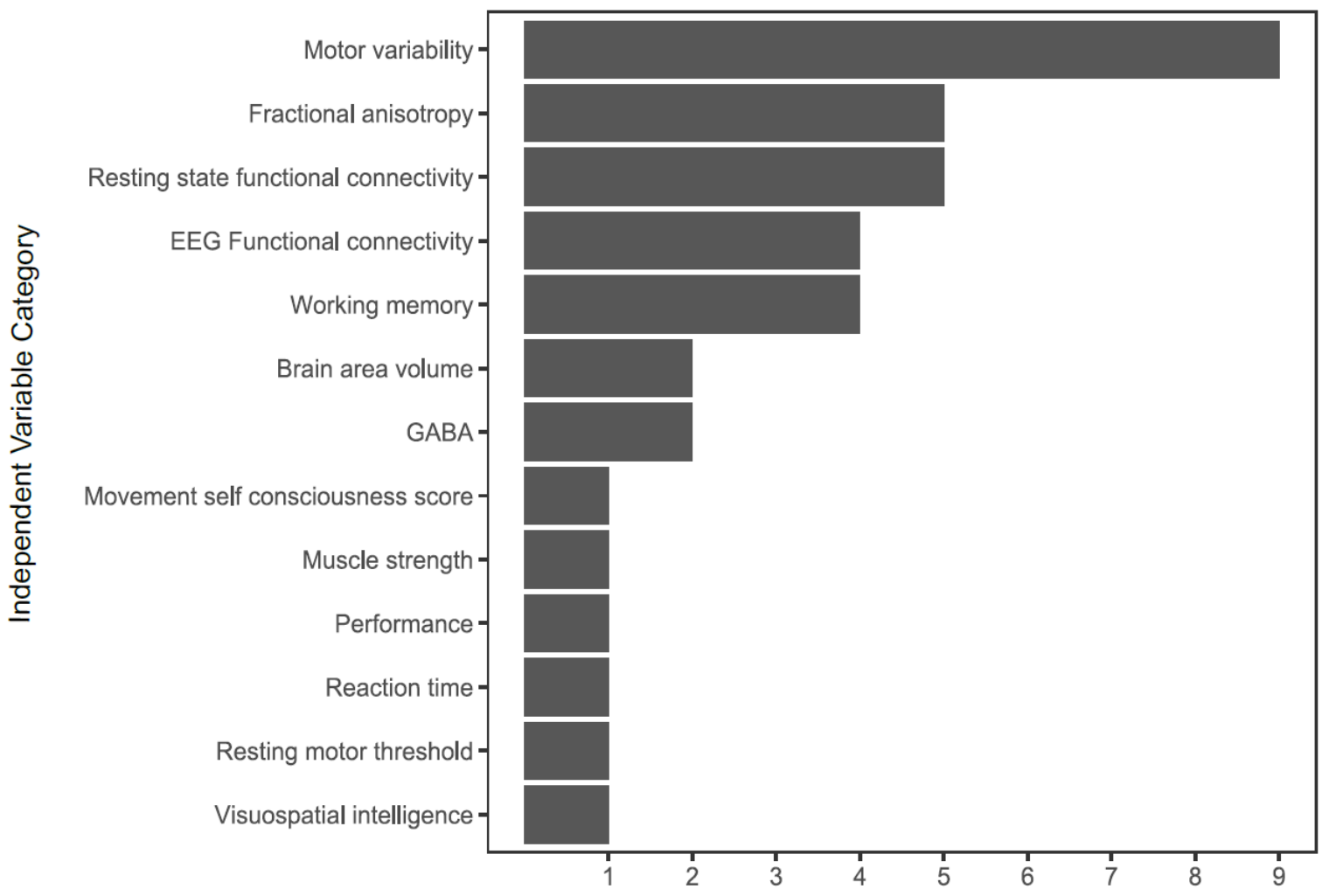

Figure 1. Distribution of predicted and predictor variables showing large variation across studies. (A) Types of predicted variables (B) Types of predictor variables based on whether they were measured 'inside' or 'outside' the task and if they were behavioral or neural variables, (C) histogram of categories of predictor variables used across studies. 


\section{Strength of prediction}

We found that the sample size was typically small (median sample size $=20$, interquartile range 8 ). Moreover, when the absolute correlation values were plotted against the sample size, we found that smaller sample sizes tended to show higher correlations (Fig 2A). To estimate the average effect size, we ran a meta-analysis of the correlation coefficients using the MAJOR package in Jamovi. The estimated average Fisher r-to-z transformed correlation was 0.6826 (corresponding to an $r$ of 0.59 ) (Fig $2 B)$.

A

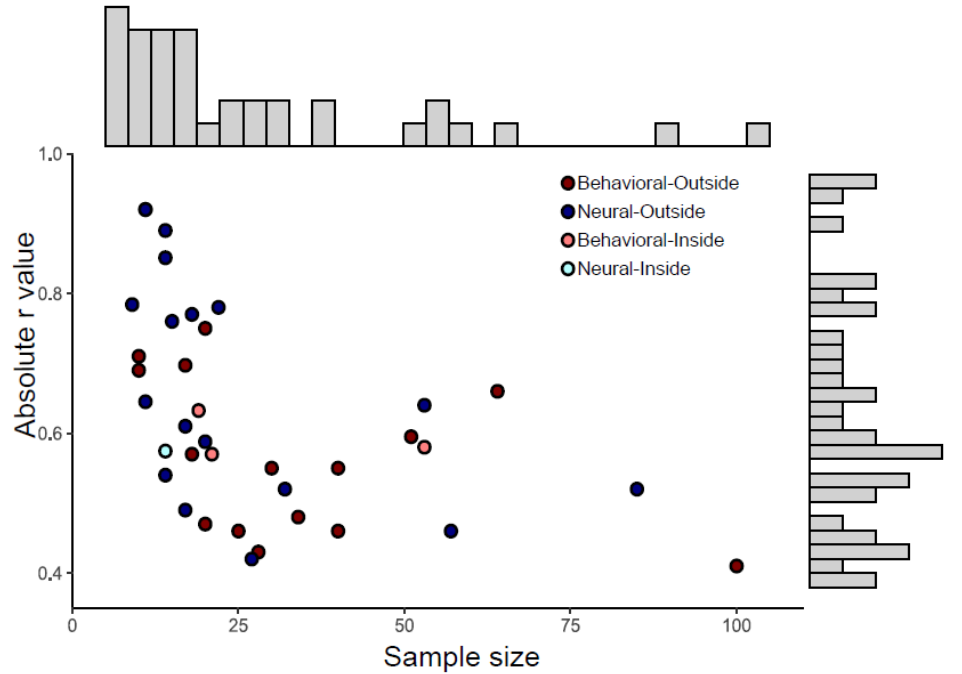

Figure 2. (A) Absolute value of Pearson's correlation coefficient (r) versus sample size. Higher sample sizes were associated with smaller correlations. Histograms of the sample size (top) and the correlation value (right) are also shown. (B) Forest plot showing Fisher r-to-z transformed correlation coefficients of individual studies plotted in increasing order and the overall estimated effect size.

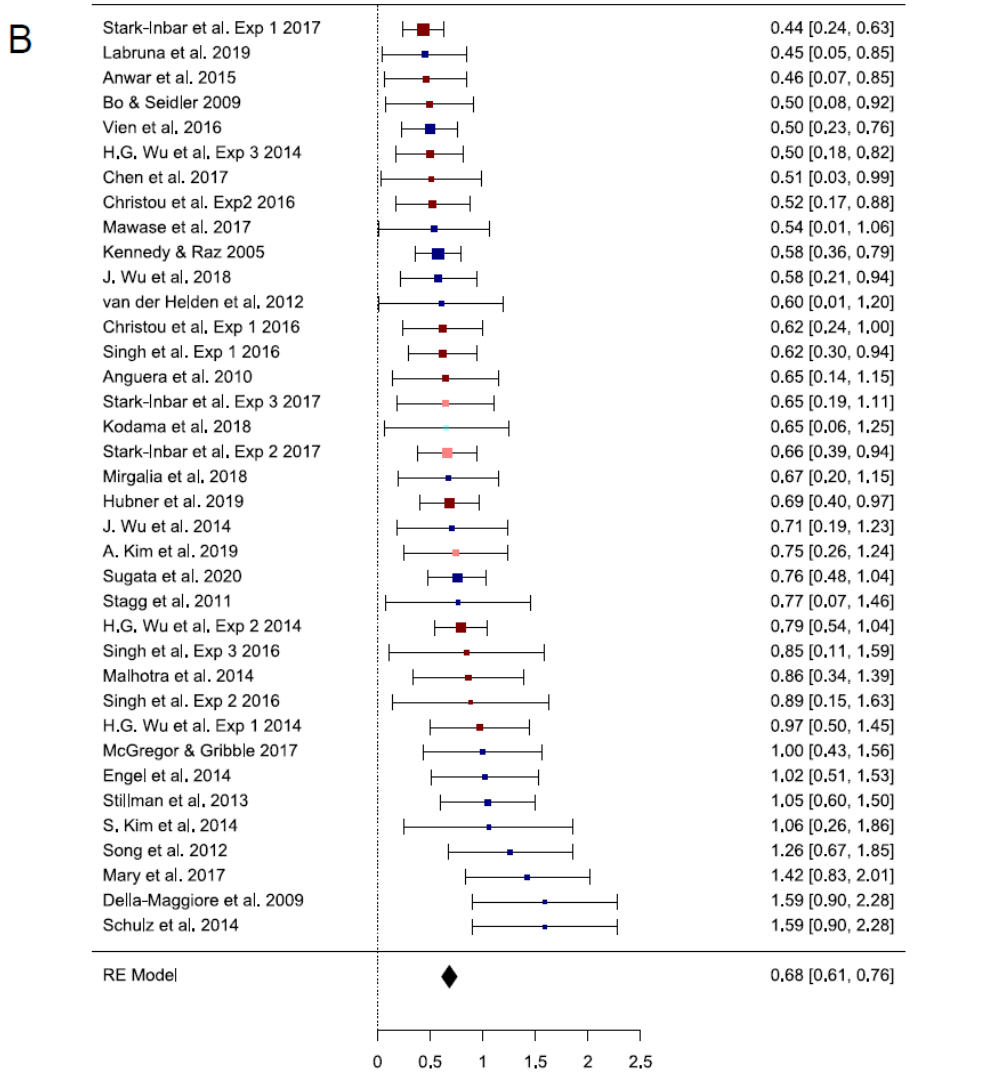




\section{Time scale of prediction}

The timescale of prediction used in studies was very short (Fig 3A). Results showed that studies used a time span of I day $(n=28,75.6 \%)$ i.e., both the predictor and predicted variable were measured on the same day). In fact, there number of studies that had a time span of 3 or more days was only $(n=$ $4,10.8 \%)$

\section{Type of motor task}

Finally, we found that an overwhelming majority of tasks (8I\%) were from just two categories (Fig $3 B)$ - adaptation $(n=18,48.6 \%)$ and sequence learning $(n=12,32.4 \%)$. Certain categories of tasks (e.g., tasks requiring the reduction of variability or the acquisition of coordination patterns) were not used.

A

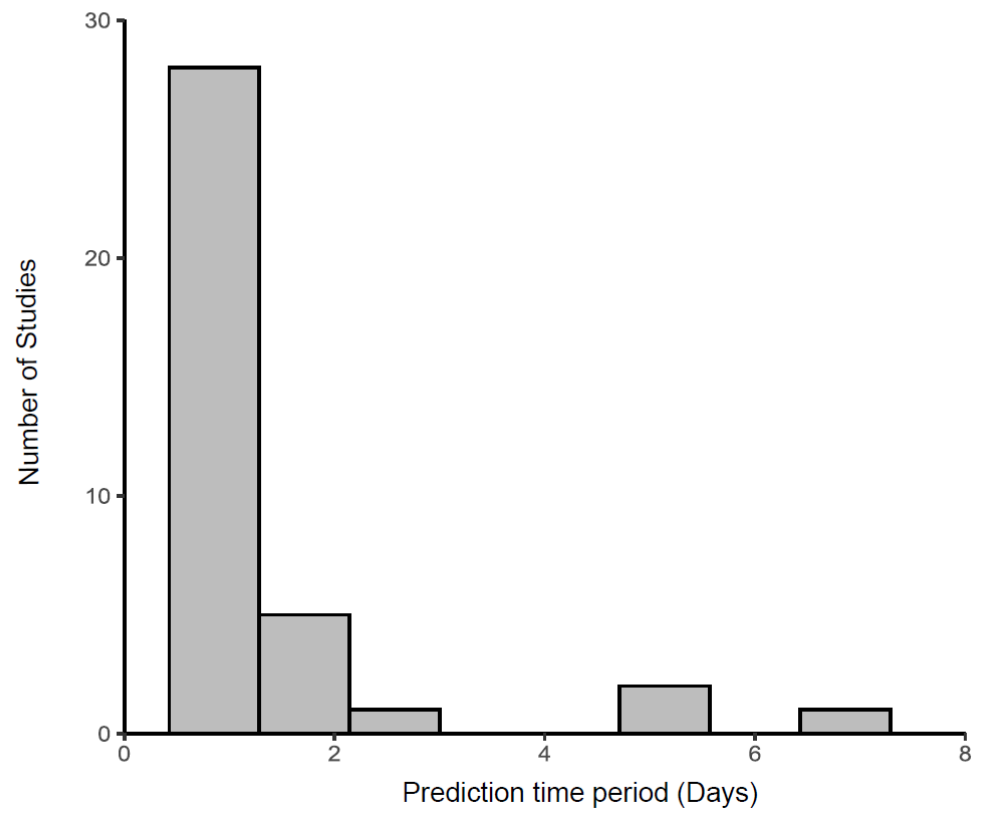

Figure 3. (A) Prediction time span and (B) Motor task type. Prediction time spans were very short, mostly confined to a single day (i.e., both predicted and predictor variables measured on the same day). A majority of studies relied on two types of tasks - adaptation and sequence learning.

B

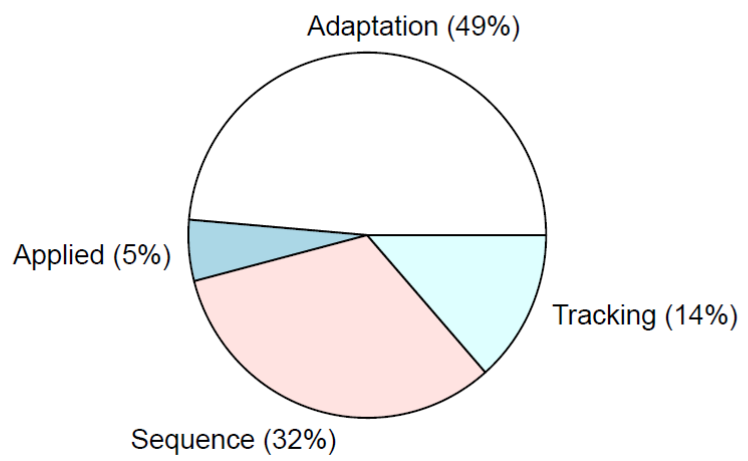




\section{Discussion}

The purpose of this review was to critically examine the evidence for predictions of individual differences in motor learning. Overall, our results highlight four critical challenges in the evidence for predicting individual differences in motor learning. First, there was a wide variation in both predicted and predictor variables across studies even when tasks were similar, indicating there is potentially a large number of 'researcher degrees of freedom' available in these studies. Second, the strength of these correlations was inversely dependent on the sample size, indicating that there is a risk of these correlations being inflated. Third, the timescale of predictions was extremely short, mostly restricted to the same day. Finally, an overwhelming majority of the studies tended to focus on two types of motor tasks - sequence learning and adaptation, indicating a gap in the field for learning other types of tasks.

In view of these challenges, we suggest the following four recommendations for future research:

\section{Adopting pre-registration and transparent reporting}

Given the variation observed in both predictor and predicted variables, the results highlight the need for transparent reporting in studies of individual differences. Pre-registrations and registered reports can help minimize researcher degrees of freedom and reduce the chances of using undisclosed flexible analyses to find relationships (or at least clearly label such findings as exploratory) (Simmons et al., 20II; Chambers, 2013; Wicherts et al., 2016). In terms of the predictor variables in motor learning experiments, given the potential for a large number of measures at different levels of analysis (e.g., movement outcome, kinematics, coordination, neural), pre-registration can help reduce selection bias in the independent variables used for prediction.

Pre-registration can also reduce arbitrariness in the dependent variables related to motor learning. We found a large variation in not only the learning measure (e.g., learning level or learning rate), but even within the same type, there were differences in how they were operationalized. For example, we found especially that "rates of learning" or "change scores" were operationalized differently across studies. It is critical to note that some measures of learning like change scores may be misleading in certain contexts and can create correlations because of statistical artifacts (Schmidt, 1972; Hawe et al., 2018; Anderson et al., 202I). While it is important to acknowledge that learning measures are not 'one-size-fits-all' and that the exact learning measure or function for the learning curve will depend on the task and context (Newell et al., 2006; Liu and Newell, 20I5), pre-registration can provide greater transparency surrounding the justification for specific measures.

\section{Increasing sample size}

Perhaps not surprisingly and consistent with other reviews on motor learning (Lohse et al., 2016; Ranganathan et al., 202I), sample sizes used for these correlations tended to be small, although to some degree, these studies tended to be somewhat larger than typical group-based studies on motor learning (median of 20 compared to 13 in group studies). More critically though, there was an inverse relation between the absolute value of the correlation and the sample size, which mirror similar findings in a review on social neuroscience studies (Vul and Pashler, 2017). This indicates that low sample size not only decreases power (i.e., the ability to detect an effect when it is present), but that it can also generate spuriously high correlations (loannidis, 2008; Yarkoni, 2009; Yarkoni and Braver, 2010). 
Therefore, in addition to pre-registration, larger sample sizes are needed for more robust correlational evidence (Schönbrodt and Perugini, 20I3).

\section{Increasing time scale of prediction}

The time scale of predictions here were all mostly within the same day. While we acknowledge that the timescale of prediction is not an 'absolute measure' and should be considered in context of the time scale of the learning process (e.g., the timescale of prediction for a task takes a few minutes to learn is not directly comparable to a task that takes days or weeks to learn), increasing the time scale of predictions is still critical from both methodological and theoretical reasons. From a methodological standpoint, increasing the time scale of predictions beyond a single day minimizes the effect of temporary confounding factors (e.g., fatigue or motivation) that can create issues in distinguishing between 'learning' and 'performance' (Salmoni et al., 1984; Kantak and Winstein, 2012). From a theoretical standpoint, expanding the timescale of learning over longer time scales may also allow the test of other hypotheses such as whether: (i) initial skill and final skill have differing contributions of abilities (Fleishman and Rich, 1963), (ii) individual differences can be separated into component processes that are related to the rate of learning and final levels of performance (Jones, 1970) and (iii) individual differences tend to diverge or converge with learning (Ackerman, 1987).

\section{Broaden range of tasks}

Finally, the fact that the majority of tasks used were adaptation and sequence learning may be a consequence of their popularity in the motor learning literature, especially in terms of their convenience for neuroimaging paradigms. However, using convenience as the sole metric for the choice of tasks can lead to a distorted picture of how these results have relevance to real-world learning. Several commentaries have emphasized that motor tasks are not interchangeable and that there may be fundamental differences in how certain types of tasks are learned (Newell, 1991; Bastian, 2008; Krakauer and Mazzoni, 20l I). Therefore, the use of tasks that capture other core aspects of motor learning (e.g., acquiring novel coordination patterns or reducing motor variability) may be important to test the generalizability of these ideas (Anderson et al., 202I).

However, it is important to note that the goal of broadening the types of tasks has to be balanced with the concern for 'task fragmentation' (Ranganathan et al., 202I), where too many different tasks are used making it difficult for comparing results across studies. In our view, tasks have to be chosen carefully so that they can be representative of several different types of learning, and where the properties of learning can be assessed carefully. For example, in our review, we found only a single study that quantified the reliability of the learning measures used in the task (Stark-Inbar et al., 2017) which is critical for establishing individual differences. These competing demands highlight the need for developing 'model tasks' for different domains of learning where the diversity in experimental paradigms can also be coupled with experimental rigor (Ranganathan et al., 202I).

\section{Conclusion}

In summary, the issue of predicting individual differences remains a core issue for motor learning yet there are several challenges in interpreting the current evidence for predicting individual differences. Although many of the issues raised have been highlighted in other reviews (Seidler and Carson, 
2017; Vul and Pashler, 2017; Anderson et al., 2021), it is clear that the recommendations needed to address these challenges (e.g., increasing sample size, developing model tasks etc.) require the need for large scale collaborative efforts like those that have started in other domains - e.g., Psych Science Accelerator (Moshontz et al., 20I8) and ManyLabs projects (Klein et al., 2014). We anticipate that addressing these steps as a field can help provide a more robust evidence base for the study of individual differences in motor learning.

\section{Acknowledgment}

This material is based upon work supported by grant NSF 1823889

\section{Supplementary Data}

The table of individual studies used for this review and the associated references can be found at https://osf.io/7cm2y/ 


\section{References}

Ackerman PL (1987) Individual differences in skill learning: An integration of psychometric and information processing perspectives. Psychol Bull 102:3-27.

Ackerman PL (1988) Determinants of individual differences during skill acquisition: Cognitive abilities and information processing. J Exp Psychol Gen II7:288-318.

Adams JA (1987) Historical review and appraisal of research on the learning, retention, and transfer of human motor skills. Psychol Bull 10I:4I-74.

Altman DG, Royston P (2006) The cost of dichotomising continuous variables. BMJ 332:1080.

Anderson DI, Lohse KR, Lopes TCV, Williams AM (202I) Individual differences in motor skill learning: Past, present and future. Hum Mov Sci 78:1028।8.

Bastian AJ (2008) Understanding sensorimotor adaptation and learning for rehabilitation. Curr Opin Neurol 21:628-633.

Chambers CD (2013) Registered reports: a new publishing initiative at Cortex. Cortex J Devoted Study Nerv Syst Behav 49:609-610.

Cronbach LJ (1957) The two disciplines of scientific psychology. Am Psychol 12:67I-684.

Fleishman EA, Rich S (1963) Role of kinesthetic and spatial-visual abilities in perceptual-motor learning. J Exp Psychol 66:6-II.

Hands B, Mclntyre F, Parker H (2018) The General Motor Ability Hypothesis: An Old Idea Revisited. Percept Mot Skills 125:213-233.

Hawe RL, Scott SH, Dukelow SP (2018) Taking Proportional Out of Stroke Recovery. Stroke 50:204211.

Henry F M (1968) Specificity vs. Generality in Learning Motor Skill. In: Classical Studies on Physical Activity (Brown RC, Kenyon GS, eds), Pp 33I-340. Englewood Cliffs, NJ: Prentice Hall.

loannidis JPA (2008) Why most discovered true associations are inflated. Epidemiol Camb Mass 19:640-648.

Jones MB (1970) A two-process theory of individual differences in motor learning. Psychol Rev 77:353-360.

Kantak SS, Winstein CJ (2012) Learning-performance distinction and memory processes for motor skills: a focused review and perspective. Behav Brain Res 228:219-23I.

Klein RA et al. (2014) Investigating Variation in Replicability. Soc Psychol 45: 142-152.

Krakauer JW, Mazzoni P (20II) Human sensorimotor learning: adaptation, skill, and beyond. Curr Opin Neurobiol 21:636-644.

Liu Y-T, Newell KM (2015) S-Shaped motor learning and nonequilibrium phase transitions. J Exp Psychol Hum Percept Perform 4I:403-4I4.

Lohse K, Buchanan T, Miller M (2016) Underpowered and overworked: Problems with data analysis in motor learning studies. J Mot Learn Dev 4:37-58.

MacCallum RC, Zhang S, Preacher KJ, Rucker DD (2002) On the practice of dichotomization of quantitative variables. Psychol Methods 7:19-40.

Marteniuk RG (1974) Individual differences in motor performances and learning. Exerc Sport Sci Rev 2:103-130.

Moshontz H et al. (2018) The Psychological Science Accelerator: Advancing Psychology Through a Distributed Collaborative Network. Adv Methods Pract Psychol Sci I:50I-5I5.

Newell KM (199I) Motor skill acquisition. Annu Rev Psychol 42:213-237. 
Newell KM, Mayer-Kress G, Liu Y-T (2006) Human learning: Power laws or multiple characteristic time scales? Tutor Quant Methods Psychol 2:66-76.

Ranganathan R, Tomlinson AD, Lokesh R, Lin T-H, Patel P (202I) A tale of too many tasks: task fragmentation in motor learning and a call for model task paradigms. Exp Brain Res 239:1-19.

Salmoni AW, Schmidt RA, Walter CB (1984) Knowledge of results and motor learning: A review and critical reappraisal. Psychol Bull 95:355-386.

Schmidt RA (1972) The Case Against Learning and Forgetting Scores. J Mot Behav 4:79-88.

Schönbrodt FD, Perugini M (2013) At what sample size do correlations stabilize? J Res Personal 47:609-612.

Seashore RH (1930) Individual differences in motor skills. J Gen Psychol 3:38-66.

Seidler RD, Carson RG (2017) Sensorimotor Learning: Neurocognitive Mechanisms and Individual Differences. J NeuroEngineering Rehabil I4:74.

Simmons JP, Nelson LD, Simonsohn U (20I I) False-Positive Psychology: Undisclosed Flexibility in Data Collection and Analysis Allows Presenting Anything as Significant. Psychol Sci 22:1359-1366.

Stark-Inbar A, Raza M, Taylor JA, Ivry RB (2017) Individual differences in implicit motor learning: task specificity in sensorimotor adaptation and sequence learning. J Neurophysiol 1 17:4 12-428.

Vul E, Pashler H (2017) Suspiciously high correlations in brain imaging research. Psychol Sci Scrut Recent Chall Propos Solut: 196-220.

Wicherts JM, Veldkamp CLS, Augusteijn HEM, Bakker M, van Aert RCM, van Assen MALM (20I6) Degrees of Freedom in Planning, Running, Analyzing, and Reporting Psychological Studies: A Checklist to Avoid p-Hacking. Front Psychol 7:1832.

Yarkoni T (2009) Big Correlations in Little Studies: Inflated fMRI Correlations Reflect Low Statistical Power-Commentary on Vul et al. (2009). Perspect Psychol Sci 4:294-298.

Yarkoni T, Braver TS (2010) Cognitive neuroscience approaches to individual differences in working memory and executive control: Conceptual and methodological issues. In: Handbook of individual differences in cognition: Attention, memory, and executive control, pp 87-107 The Springer series on human exceptionality. New York, NY, US: Springer Science + Business Media. 